\title{
Trajectories of callous-unemotional traits from childhood to adolescence in referred youth with a disruptive behavior disorder who received intensive multimodal therapy in childhood
}

This article was published in the following Dove Press journal:

Neuropsychiatric Disease and Treatment

\author{
Gabriele Masi' \\ Simone Pisano ${ }^{2}$ \\ Paola Brovedani' \\ Gioia Maccaferri ${ }^{1}$ \\ Azzurra Manfredi' \\ Annarita Milone' \\ Annalaura Nocentini ${ }^{3}$ \\ Lisa Polidori' \\ Laura Ruglioni' \\ Pietro Muratori' \\ 'IRCCS Fondazione Stella Maris, \\ Scientific Institute of Child Neurology \\ and Psychiatry, Calambrone, Italy; \\ ${ }^{2}$ Clinic of Child and Adolescent \\ Neuropsychiatry, Department of \\ Medicine and Surgery, University of \\ Salerno, Baronissi, Italy; ${ }^{3}$ Department \\ of Sciences of Education and \\ Psychology, University of Florence, \\ Florence, Italy
}

Purpose: Our aims were to explore the developmental trajectories of callous-unemotional (CU) traits using a growth curve analysis in Italian children with disruptive behavior disorders treated with a multimodal intervention, and to test both predictors and distal outcomes of CU traits trajectories.

Patients and method: One hundred and sixty-eight children were enrolled, of whom 24 were lost in the follow-up and 144 were followed up from ages 8-9 to 14-15 years with four assessment points. Patients included 128 males $(88.9 \%)$ with a mean age of 8.7 years, 96 with oppositional defiant disorder (66.7\%) and 48 with conduct disorder (CD) (33.3\%). The developmental trajectories of CU traits were assessed with the Inventory of Callous-Unemotional Traits (ICU).

Results: Our findings revealed that CU features were likely to fit a quadratic model from childhood to adolescence. The CU traits tended to decrease during childhood, with stabilization in adolescence and a significant variability in the growth curves. Pretreatment $\mathrm{CD}$ and higher levels of externalizing behavioral problems were associated with higher level of CU traits at baseline, whereas positive parenting was associated with lower levels. No significant effects were found for all the other predictors (socioeconomic status, negative parenting, combined pharmacotherapy). Regarding outcomes into adolescence, both higher levels of CU traits at the baseline and a lower decrease of $\mathrm{CU}$ traits across time points predicted a higher risk of CD diagnosis, and higher rate of referrals to mental health services and of substance use. Furthermore, pretreatment CD and negative parenting predicted a higher risk of substance use into adolescence.

Conclusion: Our findings suggest that a close monitoring of CU traits in referred children with disruptive behavior disorders may help to detect the patients at higher risk of poor outcome.

Keywords: conduct disorder, oppositional defiant disorder, callous-unemotional traits, aggressive behavior, child psychiatry, psychopathy

\section{Introduction}

Disruptive behavior disorders (DBDs) are severe mental disorders involving deficits in regulating emotions and behavior. Given the high heterogeneity of DBD symptoms, disentangling DBD subtypes according to clinically meaningful psychopathological dimensions can improve the clinicians' diagnostic, prognostic, and therapeutic abilities. Callous-unemotional (CU) traits are the childhood precursors of the affective dimension of psychopathy, and define a subgroup of DBD youths, in terms of etiological risk factors and psychopathological features. ${ }^{1-3}$ Children with CU traits show lower levels of social competence skills, ${ }^{4}$ and adolescents with CU traits and conduct disorder (CD)
Correspondence: Pietro Muratori IRCCS Fondazione Stella Maris, Scientific Institute of Child Neurology and Psychiatry, Vialedel Tirreno 33I, 56018 Calambrone, Pisa, Italy

Tel +3950886 II I

Fax +3950886247

Email pmuratori@fsm.unipi.it 
are consistently at higher risk of behavioral and contextual problems. ${ }^{5}$ Children with a DBD diagnosis and higher levels of CU traits have an increased risk of maintaining disruptive behavior over time, ${ }^{6}$ with higher costs for the community. ${ }^{7}$

Based on this evidence, CU traits have been included in the Diagnostic and Statistical Manual of Mental Disorders, Fifth Edition (DSM-5) as part of a new specifier in the diagnostic criteria of $\mathrm{CD} .{ }^{8}$ To fit the criteria for this CD specifier, patients must present at least two features among the following: lack of remorse or guilt, callousness-lack of empathy, unconcern about performance, and shallow or deficient affects. ${ }^{8}$ These features must be present for at least 12 months, in more than one relationship and setting, according to multiple sources of information. Previous studies suggested that CU traits are relatively stable across childhood, and from childhood to adolescence. ${ }^{9}$ Overall, estimates of CU traits stability were comparable to those reported for other psychopathological constructs. ${ }^{10}$

Several studies investigated the stability of CU traits using person-centered approaches. These studies explored the relationships between variability of CU traits over time and negative distal outcomes. Fontaine et al, ${ }^{11}$ using teachers' rating of $\mathrm{CU}$ traits in a large sample of community children, found that a significant decrease in CU traits across development was more common than an increase, and they highlighted that children with high or increasing levels of CU traits presented worst outcomes at 12 years. Baskin-Sommers et al, ${ }^{12}$ using self-reported rating of CU traits in a high-risk adolescents sample, indicated that the "high" CU traits trajectory, particularly in the presence of elevated levels of conduct problems, was related to higher violence and substance use, over and above a variety of environmental risk factors among at-risk male adolescents. Klingzell et $\mathrm{al}^{13}$ investigated joint trajectories of CU traits during early childhood in a community sample using parents' reported data, and found a close relationship between CU traits and change in conduct problems. Salihovic et al ${ }^{14}$ explored the stability of psychopathic traits (including CU traits) in a sample of low-risk adolescents, and found that adolescents with persisting high levels of CU traits also maintained the highest levels of delinquency. Recently, Hawes et $\mathrm{al}^{15}$ collected parents' and teachers' reported data on a cohort of boys with an oversampling of youth exhibiting elevated conduct problems. Their data suggested that, in a sample of high-risk children, the levels of CU traits predicted the development of the interpersonal/affective features of adult psychopathy. Byrd et $\mathrm{al}^{16}$ examined distinct patterns of $\mathrm{CU}$ traits across childhood and adolescence in an at-risk sample of boys, using teachers' reported data of CU traits. According to their findings, boys in the early-onset chronic group were characterized by the highest number of risk factors. Ezpeleta et $\mathrm{al}^{17}$ examined the course of $\mathrm{CU}$ traits in a longitudinal community sample of preschoolers, using parents' and teachers' rating of CU traits. They found that CU traits predicted worse outcomes, especially when associated with anxious problems in children.

Besides investigating CU traits trajectories, several previous studies highlighted the role of several predictors in shaping CU traits trajectories. ${ }^{18,19}$ All the previous studies indicating $\mathrm{CU}$ traits as a primary target of intervention were based on community or at-risk samples. In these samples, as well as in forensic samples, it is usually difficult to gather reliable information about any kind of intervention received by the participants. We aimed to extend the exploration of predictors of levels of CU traits from the epidemiological to the clinical realm. To date, there is no information about the stability of CU traits and the severity of distal outcomes in clinical samples using a person-oriented approach such as growth curve modeling. ${ }^{1}$ Moreover, from a clinical standpoint, it is important to test whether the effects of CU traits on outcomes are affected by other clinical characteristics. Different predictors may play a specific role and load differently in severely impaired patients compared to community samples.

We also investigated whether CD diagnosis and combined pharmacotherapy during childhood may affect CU traits trajectories or predict adolescent outcomes. It is established that $\mathrm{CD}$ diagnosis is associated with higher levels of $\mathrm{CU}$ traits, ${ }^{20}$ and with worse long-term prognosis, such as substance abuse or need for mental health assistance. ${ }^{21}$ Adding pharmacotherapy among the predictors may help to test a possible effect of medications on CU traits trajectories, ${ }^{22}$ and control for the effect of drugs on outcomes. ${ }^{23}$

In addition, we extended the exploration on possible predictors of distal outcome, by including parenting ${ }^{24}$ and socioeconomic status (SES). ${ }^{25}$ Understanding the role of specific parenting components in CU traits trajectories may be relevant for identifying possible treatment strategies for children with DBDs and high levels of CU traits. Several studies have examined the influence of parenting on the development of CU traits. Harsh parenting practices have been associated with behavioral problems in youths with low levels of CU traits; ${ }^{26}$ however, previous studies indicated that parenting might directly promote CU traits themselves. ${ }^{27}$ Previous studies suggested that parental warmth might be the parenting dimension more negatively associated with high and stable levels of CU traits in children. ${ }^{28,29}$ On the contrary, Viding et $\mathrm{al}^{4}$ found that parenting is not a predictor of the levels of CU traits in children. However, more studies are needed to test the unique 
effect of different aspects of parenting (negative or positive) in predicting $\mathrm{CU}$ traits trajectories in a clinical sample.

In a previous pilot study, we used the Antisocial Process Screening Device (APSD) parent report to investigate trajectories of CU traits in DBD children who had received multimodal treatment and were followed up until adolescence. ${ }^{28}$ Through a latent growth curve model, we found that $\mathrm{CU}$ traits tended to decrease until adolescence, with subsequent stabilization, and that a slower decrease of CU traits was related to more severe outcomes during adolescence. However, the study presented several limitations, particularly the relatively small sample size $(\mathrm{N}=59)$ and the use of APSD CU subscale thr psychometric proprieties of which are not strongly validated..$^{30}$

In the current study, we analyze the latent growth curves of CU traits in a larger sample $(\mathrm{N}=144)$ of referred children, using a self-report measure, the total score from the self-report Inventory of Callous-Unemotional Traits (ICU) (Frick PJ. The Inventory of Callous-Unemotional Traits. Unpublished rating scale. The University of New Orleans; 2003). We maintain that this self-report measure may be a more sensitive instrument to investigate $\mathrm{CU}$ traits within research conditions of complete confidentiality. ${ }^{31}$ The ICU is characterized by acceptable internal consistency, as well as by an easier translation of its items in the DSM-5 CU specifier, as they were used in the secondary data analyses that guided the DSM-5 specifier formation. ${ }^{32}$ Furthermore, the four DSM-5 criteria symptoms for the specifier were based on the original four items that guided the item pool for developing the ICU.

The main aim of this study was to determine the trajectory of the self-report CU traits in patients with DBDs during a 1-year multimodal treatment period (children aged 8-9 years of age) and at two follow-ups up until adolescence (ages 14-15 years), in order to explore the changeability of CU traits. We also explored the ability of the time-invariant predictors at baseline to predict the intercept and change components, as well as the ability of the intercept and change components to predict the time-invariant outcomes into adolescence. Predictors included CD diagnosis, levels of externalizing behavioral problems, parenting variables, and combined pharmacotherapy. We hypothesized that high and persistent CU traits from childhood to adolescence are associated with poorer outcomes. Selected negative outcomes in adolescence were diagnosis of $\mathrm{CD}$, degree of substance use, and frequency of mental health referrals during the previous 12 months.

\section{Methods}

\section{Participants and procedure}

This study is based on a sample of patients referred to our psychiatric hospital. Child psychiatrists interviewed children and parents, using the Schedule for Affective Disorders and Schizophrenia for School-Age Children Present and Lifetime Version (K-SADS-PL). ${ }^{33}$ The Wechsler Intelligence Scale for Children - third edition ${ }^{33}$ was used to evaluate IQ. One hundred and sixty-eight children met the following inclusion criteria: 1) main diagnosis of oppositional defiant disorder (ODD) or conduct disorder (CD) according to K-SADS-PL; $; 4$ and 2) a full-scale IQ of 85 or above. Of the patients who met the inclusion criteria, 24 were lost in the follow-up and the remaining 144 were included in the study. These patients, with a mean age of 8.7 years, included 128 boys $(88.9 \%)$ and 16 girls (11.1\%), 96 (66.7\%) with ODD, 48 (33.3\%) with CD, and 30 (20.8\%) with comorbid attention-deficit/ hyperactivity disorder, and were assessed at each follow-up. Regarding family SES, assessed with the Hollingshead and Redlich categories, ${ }^{35} 39$ (27.1\%) of the families had low SES, $71(49.3 \%)$ had medium SES, and 34 (23.6\%) had high SES. No significant differences were found from attrition analysis in age, gender, diagnosis ratio (ODD/CD), SES, and level of CU traits between those who dropped out and those who completed the treatment.

Children received a psychotherapeutic intervention based on cognitive behavioral techniques. All the children started the treatment when they were 8-9 years old, and for all the children, the treatment lasted 15 months. Treatment was organized in weekly sessions, and included individual psychotherapy for children and individual parent training. Individual therapy was focused on teaching children to improve self-control, problem-solving skills, and perspective taking. We used the following evidence-based practices to reach these goals: goal setting, modeling, positive reinforcements, anger coping techniques, role playing, and homework. During individual parent training, strategies (such as correct use of prize, response cost, ignoring, and time-out) were introduced. The average child and parent attendance rate in the current sample was $87 \%$. Several measures were adopted to assure the best clinical standard: 1) therapists attended official training in cognitive behavioral psychotherapy; 2) therapists attended supervision meetings with a certified cognitive behavioral psychotherapy supervisor; and 3) a weekly staff meeting, based on case reviews, was performed in order to complete a checklist of objectives delivered within sessions and to monitor treatment adherence. All the therapists involved in the current study had a master's degree in psychology and attended official training in psychotherapy, as required by the Italian law. Forty-eight patients (33\%) received an additional pharmacotherapy: 20 methylphenidate, 18 an antipsychotic (AA), and 10 a mood stabilizer (STAB). 
The participants were $8-9$ years old at the first evaluation point, and they were 14-15 at the last evaluation point. We tested children and parents at Time (T) 1=ages 8-9 (pretest), T2=18 months after the pretest (posttest 1 ), $\mathrm{T} 3=34-36$ months after the pretest (posttest 2 ), and T4=70-72 months after the pretest (posttest 3). All subjects (children and parents) gave written informed consent in accordance with the Declaration of Helsinki. The protocol was approved by the Ethical Committee of IRCCS Fondazione Stella Maris.

\section{Measures}

\section{Child diagnosis}

We used the K-SADS-PL which is an interview according to Diagnostic and Statistical Manual of Mental Disorders, Fourth Edition ${ }^{36}$ that explores the presence or absence of each of the symptoms in different psychiatric syndromes. Trained child psychiatrists with specific experience in adolescent psychiatric disorders administered the clinical interview to the child and to the mother.

\section{Inventory of Callous-Unemotional Traits}

It is a 24-item measure administered to patients at four time intervals during the study, and designed to assess $\mathrm{CU}$ traits in youth (Frick PJ. The Inventory of Callous-Unemotional Traits. Unpublished rating scale. The University of New Orleans; 2003). The total score of ICU - self report was used to evaluate $\mathrm{CU}$ traits in children across time in the current sample. Patients were asked to indicate how much they agreed with every item (eg, The feelings of others are unimportant to me, I try not to hurt others' feelings; I hide my feelings from others), using a 4-point Likert-type scale, from not at all true (0) to definitely true (3). In Italian preadolescents, a three-factor model (callousness, uncaring, and unemotional) loads on a general callous-unemotional factor. ${ }^{37}$ The internal reliability of the total CU scale in the current sample was 0.74 at T1, 0.75 at T2, 0.71 at T3, and 0.78 at T4 (Cronbach's alpha).

\section{Pretreatment predictors}

All of the following measures were assessed at baseline.

\section{Categorical diagnosis}

We used the clinical interview K-SADS-PL. ${ }^{34}$ The rate of patient-parent K-SADS diagnosis agreement was $89 \%$. The predictor was the presence of a pretreatment $\mathrm{CD}$ diagnosis.

\section{Externalizing behavioral problems}

We used the externalizing domain of the Child Behavior Check List ${ }^{38}$ completed by parents. The reliability coefficient (Cronbach's alpha) was 0.83 .

\section{Negative or positive parenting practices}

The Alabama Parenting Questionnaire (APQ) ${ }^{39}$ - mother report was used to evaluate parenting practices with five subscales: parental involvement, positive parenting, poor monitoring/supervision, inconsistent discipline, and corporal punishment. Items (eg, You have a friendly talk with your child; You praise your child; Your child is not punished when he/she has done something wrong) are rated on a 5-point Likert scale, ranging from 1 (never) to 5 (always). We derived two parenting composites: a positive parenting composite, including the APQ parental involvement and positive reinforcement subscales; and a negative parenting composite, including APQ inconsistent discipline and corporal punishment subscales. ${ }^{40}$ In the current study, we used these two components as predictors. The reliability coefficients (Cronbach's alpha) were 0.85 for positive parenting composite and 0.82 for negative parenting composite.

\section{Socioeconomic status}

The Hollingshead and Redlich categories ${ }^{35}$ define five class levels, considering education and occupation of the family, as well as location of the family place of residence.

\section{Use of pharmacotherapy}

We used clinical records report on the use of psychopharmacological intervention. The predictors were the different types of pharmacological treatment that were prescribed. Each type of pharmacological treatment was a dichotomous variable.

\section{Adolescence outcome measures}

All of the following measures were assessed at the final assessment point.

\section{Categorical diagnosis}

We used the clinical interview K-SADS-PL. ${ }^{34}$ The interviewers were blind to the objectives of the current research. The rate of child-parent K-SADS diagnosis agreement was $86 \%$. $\mathrm{CD}$ diagnosis was used as a measure of adolescent outcome (dichotomous variable).

\section{Substance use}

The Substance Abuse Prevention (CSAP) Student Survey is a 14 -item self-report questionnaire. ${ }^{41}$ Self-report survey assessments of youths' substance use have been found to be reliable and valid. ${ }^{42}$ The CSAP items measure patients' use of alcohol, tobacco, or marijuana in the past month, and they were aggregated to produce the outcome measure. 
Table I Descriptive variables

\begin{tabular}{|c|c|c|c|c|c|c|c|c|c|c|c|c|c|c|c|}
\hline & I & 2 & 3 & 4 & 5 & 6 & 7 & 8 & 9 & 10 & II & 12 & 13 & 14 & 15 \\
\hline I. CU TI & 1.00 & & & & & & & & & & & & & & \\
\hline 2. $\mathrm{CU} \mathrm{T} 2$ & 0.79 & 1.00 & & & & & & & & & & & & & \\
\hline 3. CU T3 & 0.69 & 0.89 & 1.00 & & & & & & & & & & & & \\
\hline 4. CU T4 & 0.45 & 0.47 & 0.55 & 1.00 & & & & & & & & & & & \\
\hline 5. SES & -0.03 & -0.04 & -0.04 & 0.01 & 1.00 & & & & & & & & & & \\
\hline 6. $\mathrm{CD}$ diagnosis $\mathrm{TI}$ & 0.30 & 0.29 & 0.35 & 0.04 & 0.12 & 1.00 & & & & & & & & & \\
\hline 7. Externalizing TI & 0.33 & 0.29 & 0.31 & 0.30 & -0.08 & 0.25 & 1.00 & & & & & & & & \\
\hline 8. AA & 0.11 & 0.08 & 0.01 & 0.18 & 0.08 & -0.03 & 0.04 & 1.00 & & & & & & & \\
\hline 9. METH & 0.00 & 0.00 & 0.04 & 0.03 & -0.21 & 0.04 & 0.07 & -0.16 & 1.00 & & & & & & \\
\hline 10. STAB & 0.21 & 0.21 & 0.23 & 0.07 & -0.07 & 0.22 & 0.11 & -0.10 & -0.09 & 1.00 & & & & & \\
\hline II. Positive parenting & -0.42 & -0.51 & -0.46 & -0.43 & 0.04 & -0.10 & -0.12 & -0.09 & 0.01 & -0.25 & 1.00 & & & & \\
\hline 12. Negative parenting & -0.01 & 0.02 & -0.03 & 0.07 & -0.01 & -0.18 & 0.05 & -0.05 & -0.01 & 0.03 & -0.16 & 1.00 & & & \\
\hline 13. CD diagnosis $\mathrm{T} 4$ & 0.46 & 0.48 & 0.58 & 0.48 & 0.18 & 0.38 & 0.16 & 0.09 & -0.13 & 0.28 & -0.30 & -0.01 & 1.00 & & \\
\hline 14. Substance use T4 & 0.35 & 0.57 & 0.62 & 0.66 & 0.08 & 0.24 & 0.15 & 0.23 & -0.05 & 0.08 & -0.37 & 0.12 & 0.53 & 1.00 & \\
\hline I5. MH T4 & 0.10 & 0.08 & 0.13 & 0.44 & 0.13 & 0.00 & 0.16 & 0.36 & 0.08 & -0.01 & -0.15 & 0.10 & 0.14 & 0.35 & 1.00 \\
\hline Mean & 27.583 & 23.974 & 21.545 & 23.024 & 2.826 & 1.252 & 70.159 & 0.159 & 0.121 & 0.056 & 58.617 & 21.052 & 0.103 & 5.917 & 0.171 \\
\hline SD & 5.38 & 6.11 & 6.32 & 6.58 & 0.70 & 0.43 & 4.79 & 0.036 & 0.033 & 0.023 & 5.61 & 3.27 & 0.33 & 2.38 & 0.388 \\
\hline
\end{tabular}

Notes: Time (T) I= ages 8-9 (pretest), T2=18 months after the pretest (posttest I), T3=34-36 months after the pretest (posttest 2), and T4=70-72 months after the pretest (posttest 3).

Abbreviations: $\mathrm{AA}$, antipsychotic; $\mathrm{CD}$, conduct disorder; $\mathrm{CU}$, callous-unemotional; $\mathrm{METH}$, methylphenidate; $\mathrm{MH}$, referrals to mental health services; $\mathrm{SES}$, socioeconomic status; STAB, mood stabilizer.

\section{Referrals to mental health services}

At the adolescence follow-up, clinicians interviewed parents about the frequency with which they had used mental health services: "How many times during the last 12 months did you refer to a hospital or day treatment unit for behavioral or emotional problems of your child?"

\section{Statistical analyses}

Two models were tested and compared with each other: a linear model and a quadratic model. A linear model assumes that the development of $\mathrm{CU}$ traits is linear across time and represents a constant change over time (ie, CU traits would decrease or increase constantly across time). A quadratic model assumes that the development is not linear (ie, CU traits would decrease or increase for certain groups of individuals after a period of decrease or increase). In all the models tested, we defined the intercept as CU traits at age 9, and the factor loadings for the slope were set to $0,1,2$, and 4 . Then, a conditional model was estimated where the unconditional model was extended including the main effects of time-invariant variables and the outcomes in adolescence. The latent growth factors with significant variability around the mean were regressed on the background variables, and the outcome constructs were simultaneously regressed into the $\mathrm{CU}$ traits intercept and the $\mathrm{CU}$ traits linear and quadratic slope factors, as well as all the predictors. Table 1 shows the descriptive variables of our sample. All the analyses were conducted using Mplus 7. ${ }^{43}$ Maximum likelihood estimator was used. To avoid bias due to the limited attrition in the sample, we estimated all models using the direct maximum likelihood procedure available in Mplus. Model fit was evaluated using the maximum likelihood ratio test statistic $\left(\chi^{2}\right)$, the root mean square error of approximation (RMSEA), and the comparative fit index (CFI). Recommended cutoff points for these measures are $0.08^{44}$ or $0.06^{43}$ for RMSEA, and 0.95 for CFI. ${ }^{45}$ The estimation and the prediction of longitudinal development of CU traits trajectory were analyzed through the latent growth curve model. ${ }^{46}$ This model can be extended in order to evaluate whether individual variability of intercept and slope can be predicted by a set of explanatory variables.

\section{Results}

\section{Unconditional latent growth curve}

We tested a linear model, but the model did not fit the data well $\left(\chi^{2}(5, \mathrm{~N} ; 144)=109.048 ; P<0.001 ;\right.$ RMSEA $=0.380$; $\mathrm{CFI}=0.650$ ). Therefore, we tested a quadratic model and the model fit the data well $\left(\chi^{2}(1, \mathrm{~N} ; 144)=0.163 ; P=0.69\right.$; RMSEA $=0.000 ; \mathrm{CFI}=1.00$ ). There was a significant mean of the intercept (intercept mean $=28.134 ; \mathrm{SE}=0.49 ; t=57.239$; $P<0.001$ ), indicating that the overall group reported a positive average starting point at age 9 , and a significant variance of the intercept (intercept variance $=22.554 ; \mathrm{SE}=4.664 ; t=4.856$; $P<0.001$ ), reflecting interindividual variability around this mean group. The mean of the slope was significant and negative (linear slope mean $=-4.933 ; \mathrm{SE}=0.431 ; t=-11.451$; $P<0.001$ ), showing on average a tendency to linearly decrease across time. A significant variance of the slope was found (linear slope variance $=13.065 ; \mathrm{SE}=4.783 ; t=2.731 ; P<0.001$ ), showing interindividual variability in growth over time. The 
correlation between latent intercept and linear slope was not significant ( $r=-0.14 ; \mathrm{SE}=3.802 ; t=-0.037 ; P=0.971$ ). Finally, the mean of the quadratic factor was significant and positive (quadratic slope mean=0.904; $\mathrm{SE}=0.103 ; t=8.773 ; P<0.001$ ), with a significant variance (quadratic slope variance $=0.774$; $\mathrm{SE}=0.259 ; t=2.998 ; P<0.010)$, showing on average a tendency to upturn from T3 to T4 beyond what is predicted by the linear decrease. In summary, CU traits showed significant decrease with the decrease becoming significantly less over time (the negative slope becomes less and less over time, until it becomes slightly positive for the last interval from T2 to T4, as shown in Figure 1).

\section{Growth model with time-invariant predictors at $\mathrm{TI}$ and outcomes in adolescence}

In the conditional model, the growth factors with significant interindividual variability around the mean were regressed on all the predictors described above, and the outcomes (CD diagnosis, risk of using substances, and rate of referrals to mental health services) were regressed on growth factors and on all the predictors (Table 2 ). The model fit the data well $\left(\chi^{2}(11, \mathrm{~N} ; 115)=23.154 ; P<0.05 ; \mathrm{RMSEA}=0.078\right.$; $\mathrm{CFI}=0.982$ ). Results showed that $\mathrm{CD}$ diagnosis was significantly related to the intercept and to the quadratic factor. In particular, the diagnosis of CD predicted higher levels of CU at T1 and a lower tendency to upturn from T3 to T4, beyond what is predicted by the linear decrease. Externalizing levels at T1 were related to the intercept factor: higher level of externalizing symptoms predicted a higher CU trait at $\mathrm{T} 1$. We found a relationship between positive parenting and $\mathrm{CU}$ trait at $\mathrm{T} 1$, indicating that higher levels of positive parenting at $\mathrm{T} 1$ are associated with lower levels of CU traits

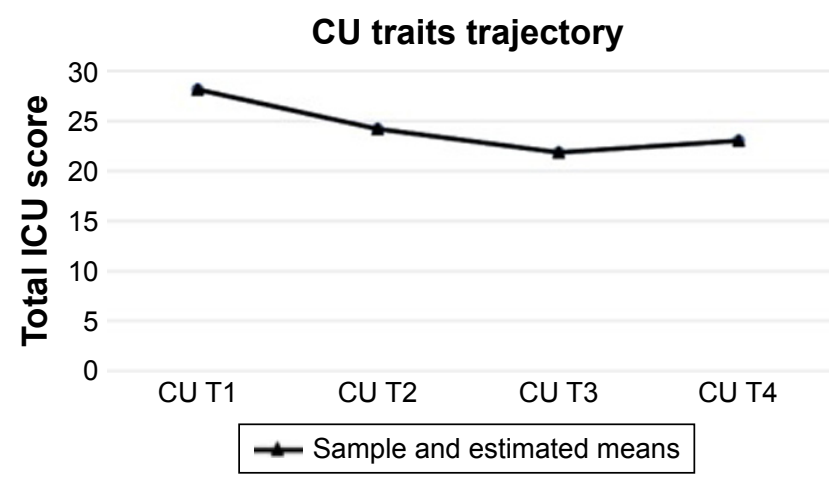

Figure I Sample and estimated means for the CU traits trajectory.

Notes: Time (T) I=ages 8-9 (pretest), T2=18 months after the pretest (posttest I), $\mathrm{T} 3=34-36$ months after the pretest (posttest 2), and T4=70-72 months after the pretest (posttest 3).

Abbreviations: CU, callous-unemotional; Inventory of Callous-Unemotional Traits.
Table 2 Conditional model with time-invariant predictors and outcomes

\begin{tabular}{|c|c|c|c|}
\hline Predi & $\begin{array}{l}\text { Intercept } \\
\text { Beta (SE) }\end{array}$ & $\begin{array}{l}\text { Linear slope } \\
\text { Beta (SE) }\end{array}$ & $\begin{array}{l}\text { Quadratic slope } \\
\text { Beta (SE) }\end{array}$ \\
\hline SES & $-0.204(0.64)$ & $-0.264(0.589)$ & $0.109(0.154)$ \\
\hline CD diagnosis & $2.037(1.080)^{\mathrm{a}}$ & $1.685(0.996)$ & $-0.613(0.26 \mathrm{I})^{\mathrm{b}}$ \\
\hline Externalizing TI & $0.260(0.094)^{b}$ & $-0.038(0.087)$ & $0.016(0.023)$ \\
\hline AA & $1.200(1.217)$ & $-0.488(1.121)$ & $0.208(0.221)$ \\
\hline METH & $-0.115(1.381)$ & $0.150(1.284)$ & $0.029(0.340)$ \\
\hline STAB & 1.405 (2.202) & $0.266(1.85 I)$ & $-0.182(0.478)$ \\
\hline Positive parenting & $-0.368(0.084)^{c}$ & $-0.119(0.078)$ & $0.023(0.021)$ \\
\hline Negative parenting & $0.048(0.143)$ & $-0.032(0.134)$ & $0.009(0.036)$ \\
\hline Prec & $\begin{array}{l}\text { CD_T4 } \\
\text { Beta (SE) }\end{array}$ & $\begin{array}{l}\text { Substance } \\
\text { Beta (SE) }\end{array}$ & $M$ \\
\hline SES & $0.070(0.038)^{\mathrm{a}}$ & 0.106 (0.229) & $0.074(0.049)$ \\
\hline CD diagnosis & $0.171(0.068)^{b}$ & $1.015(0.413)^{\mathrm{b}}$ & $0.028(0.089)$ \\
\hline Externalizing TI & $0.007(0.006)$ & $-0.055(0.037)$ & $0.005(0.008)^{b}$ \\
\hline AA & $0.020(0.077)$ & $0.799(0.468)$ & $0.346(0.101)^{c}$ \\
\hline METH & $-0.111(0.087)$ & $-0.457(0.528)$ & $0.187(0.115)$ \\
\hline STAB & $0.225(0.112)^{a}$ & $-0.447(0.687)$ & $0.07 \mid(0.147)$ \\
\hline Positive parenting & $0.004(0.006)$ & $0.011(0.037)$ & $-0.001(0.008)$ \\
\hline Negative parenting & $-0.001(0.010)$ & $0.117(0.060)^{\mathrm{a}}$ & $0.011(0.013)$ \\
\hline Intercept & $0.036(0.007)^{c}$ & $0.243(0.042)^{c}$ & $0.012(0.008)$ \\
\hline Linear slope & $0.096(0.022)^{c}$ & $1.235(0.146)^{c}$ & $0.097(0.029)^{c}$ \\
\hline Quadratic slope & $0.316(0.077)^{c}$ & $3.725(0.479)^{c}$ & $0.423(0.102)^{c}$ \\
\hline
\end{tabular}

Notes: Values are unstandardized estimates. ${ }^{a} P<0.05$; $b P<0.01 ; c P<0.001$. Time (T) $\mathrm{I}=$ ages 8-9 (pretest); T4=70-72 months after the pretest (posttest 3).

Abbreviations: $A A$, antipsychotic; $C D$, conduct disorder; $M E T H$, methylphenidate; $M H$, referrals to mental health services; $\mathrm{SES}$, socioeconomic status; $\mathrm{STAB}$, mood stabilizer.

at T1. Other predictors were not significant (SES, negative parenting component, pharmacotherapy).

Regarding the outcomes, findings indicated a significant role of intercept, linear slope, and quadratic slope in predicting $\mathrm{CD}$ diagnosis at T4 and substance use at T4, indicating that higher levels of CU traits at T1, a lower decrease from $\mathrm{T} 1$ to T3, and a higher upturn from T3 to T4 predicted a higher risk of CD diagnosis at T4 and of substance use at T4. Furthermore, results showed a significant role of linear slope and quadratic slope in predicting risk of referring to mental health services into adolescence, indicating that a lower decrease from $\mathrm{T} 1$ to $\mathrm{T} 3$ and a higher upturn from $\mathrm{T} 3$ to $\mathrm{T} 4$ predicted a higher probability of being referred to mental health services into adolescence. The presence of pretreatment $\mathrm{CD}$ diagnosis predicted the possibility of a $\mathrm{CD}$ diagnosis and of substance use in adolescence. Higher use of AA at T1 predicted a higher probability of being referred to mental health services into adolescence, and higher use of STAB predicted a higher possibility of a CD diagnosis. Higher levels of negative parenting at T1 predicted a higher level of substance use in adolescence. Finally, the model explained $49 \%$ of the variance of CD diagnosis outcome, $71 \%$ of the variance of risk of substance use outcome, and $35 \%$ of the variance of rate of referrals to mental health services. 


\section{Discussion}

At a clinical standpoint, the most important finding from the current study is that, in the context of mental health services, it is important to monitor the levels of $\mathrm{CU}$ traits in children with a DBD diagnosis, in order to detect the patients at higher risk of poorer outcome. Furthermore, $\mathrm{CU}$ traits may present a decreasing trajectory after an intensive treatment program. A primary reason for extending the construct of psychopathy from adults to youths is an increased possibility for an early and timely identification of its precursors, with all the possible diagnostic and prognostic implications. ${ }^{8}$ In this perspective, longitudinal studies with clinical samples of children with severe behavioral disorders are crucial. Furthermore, understanding whether CU traits are affected by other children's characteristics could be relevant to improve our treatment targets and strategies, as well as to plan possible prevention strategies. The present study provides clinically relevant data regarding possible trajectories of $\mathrm{CU}$ traits, investigated in children with a DBD diagnosis and treated in a hospital for child and adolescent psychiatry. Consistently with our previous report, ${ }^{28}$ the present study indicates that $\mathrm{CU}$ traits in youth receiving a specific intervention tend to decrease during childhood (across 1 year of treatment and the first year of follow-up), with a slowdown ("stabilization") during adolescence (between 12 and 15 years). Other studies support the notion that levels of CU traits may tend to decrease after children have received intervention. ${ }^{47-50}$ However, in our sample, we found a significant variability in the growth curves of $\mathrm{CU}$ traits. Our study shows that, in referred and treated children with DBDs, CU traits trajectories over time predict severe outcomes in early adolescence. Children with a DBD diagnosis and a minor decrease of CU traits, compared to those who showed a greater decrease, were at increased risk for CD diagnosis, substance use, and referral to mental health services during adolescence. These findings are consistent with previous studies, indicating that children with persistent elevated levels of CU traits during childhood are at higher risk of worse adolescent outcomes, compared with children with lower or decreasing levels of CU traits. ${ }^{51-55} \mathrm{We}$ controlled the CU effects on distal clinical outcomes for the levels of other DBD characteristics associated with more severe conditions (CD diagnosis, levels of children's externalizing behavioral problems, lower SES, negative parenting). At a clinical standpoint, it is important to note that the levels of $\mathrm{CU}$ traits during childhood remain a significant predictor for worse longitudinal outcomes, even if the levels of the other predictors used in our model are controlled for. In the light of this finding, CU symptoms should be a specific treatment target in an intervention model for children with a DBD diagnosis. Although there are several evidence-based models for reducing aggressive behavioral problems in children, ${ }^{56}$ it is not clear how to reduce the level of CU traits in children with a DBD diagnosis, even though there have been published some promising pilot studies..$^{57,58}$ The results of this study point out the importance of creating multimodal intervention models both for children with DBDs and high levels of $\mathrm{CU}$ traits, and for their parents. Furthermore, our findings, in accordance with Wymbs et al, ${ }^{55}$ may provide useful information for substance abuse prevention and interventions in at-risk youths.

The present study was also aimed at examining the role of different predictors in shaping the trajectories of $\mathrm{CU}$ traits curve. A pretreatment $\mathrm{CD}$ diagnosis was associated with a higher level of CU traits at baseline and a lower tendency to upturn across the 2-year follow-up.

In contrast to previous studies, ${ }^{26,27,29,58}$ parenting dimensions did not predict a steeper decrease of CU traits during development, and only positive parenting was associated with lower levels of CU traits in the first evaluation period. Our findings confirm that referred children with DBDs may be particularly sensitive to positive components of parenting for the development of early levels of CU traits. ${ }^{59}$ Previous studies also suggested that a lower family SES was significantly associated with higher levels of CU traits at the baseline, and a lesser decrease during childhood. ${ }^{60}$ However, in the present study, and in line with the study by Pardini et al, ${ }^{61}$ the SES was not significantly associated with elevated levels of CU traits during development. Importantly, among possible predictors of $\mathrm{CU}$ traits trajectories, pharmacological intervention resulted nonsignificant. As already reported, ${ }^{22}$ drugs are not likely to directly influence the CU traits.

Regarding other predictors of distal outcomes, we found that the presence of a $\mathrm{CD}$ diagnosis in childhood predicted its presence in adolescence, even in a model in which CU traits changes are taken into account, also supporting the chronic nature of the disorder. Previous findings ${ }^{15}$ highlighted the importance of developing targeted interventions for children with early-onset $\mathrm{CD}$, as it seems to promote the maintenance of higher levels of $\mathrm{CU}$ traits and more severe form of conduct problems into adolescence. Furthermore, higher levels of negative parenting in childhood are associated with a risk of substance use into adolescence, as already suggested. ${ }^{62}$

Finally, a pharmacological treatment with AAs at baseline predicts a higher risk to be referred to a mental health service during adolescence: this may reflect a higher baseline severity, but also the need for clinicians to constantly monitor the patients alongside the development.

We cannot extend the findings to untreated DBD populations, since the children as well as their parents were 
treated with weekly sessions for the first period of the study; also, the absence of a control comparison group does not allow us to directly test the effect of the treatment on the CU traits trajectories. However, we report on a large sample of children with a DBD diagnosis, treated in a mental health service, and the current findings suggest that monitoring the levels of CU traits of children with DBDs may help to detect the patients at higher risk of poor outcome. Children with higher levels of CU traits are at risk for substance use, CD, and referrals to mental health service into adolescence. In this context, our findings show that the self-reported version of ICU is a good instrument for identifying those children with DBDs at higher risk of more severe distal outcomes, especially when used before and after a treatment period. According to Kimonis et al, ${ }^{63}$ the self-report ICU is a valid measure to assess $\mathrm{CU}$ traits, namely under conditions of complete confidentiality, as lack of empathy and sense of guilt are emotional features less easily assessed with parentreport instruments.

A significant methodological limitation of our study is that a self-report measure was the single source of information for assessing CU traits. This issue limits possible implications of CU traits in terms of DSM-5 specifier. Combining information gleaned from the self-report ICU with other sources, that is, persons who had known the child for extended periods of time, and across relationships and settings (eg, parents, teachers, peers), may have further improved the exploration of a $\mathrm{CU}$ trait and its relation with the DSM-5 specifier. Furthermore, the sample size did not allow us to examine joint trajectories of both $\mathrm{CU}$ traits and conduct problems across a similar age period, and to explore the ability of the latent interaction of the intercept and slope factors for $\mathrm{CU}$ traits and conduct problems to predict outcomes. Also, we were not able to explore other possible predictors of CU traits, as for example, exposure to violence in childhood ${ }^{18}$ or the ODD diagnosis.

However, our study presents some meaningful clinical implications. Primarily, we describe developmental trajectories leading to severe behavioral problems in children with a DBD diagnosis. In this context, our findings (the negative linear slope) indicate that children's CU traits may decrease if treated in a timely way and intensively, and that, despite a possible decrease during and soon after the treatment, the levels of CU traits should be monitored over time to detect patients still at risk for CD and substance use into adolescence. There is some suggestion that children with high levels of CU traits may need individualized and intensive interventions to determine sustained reductions in these features over time. ${ }^{64}$ We may include in our treatment model new components in order to better target the CU traits. Considering clinical characteristics of children with elevated levels of CU traits, an emotion recognition training, an enhancing motivation component, and a further component supporting positive emotion and positive parenting may represent possible candidates to enrich our treatment strategy.

\section{Author contributions}

GM and PM designed the study with collaboration from SP. AM and PM executed the study and wrote the paper. AN analyzed the data. PM designed and executed the study. All authors contributed toward data analysis, drafting and critically revising the paper, gave final approval of the version to be published, and agree to be accountable for all aspects of the work.

\section{Disclosure}

GM was on the advisory boards for Eli Lilly, Shire, and Angelini, has received research grants from Eli Lilly and Shire, and has been a speaker for Eli Lilly, Shire, Lundbeck, and Otsuka. The authors report no other conflicts of interest in this work.

\section{References}

1. Frick PJ, Ray J, Thornton LC, Kahn RE. Can callous-unemotional traits enhance the understanding, diagnosis, and treatment of serious conduct problems in children and adolescents? A comprehensive review. Psychol Bull. 2014;140(1):1-57.

2. Pisano S, Muratori P, Gorga C, et al. Conduct disorders and psychopathy in children and adolescents: aetiology, clinical presentation and treatment strategies of callous-unemotional traits. Ital J Pediatr. 2017;43(1):84.

3. Masi G, Milone A, Pisano S, et al. Emotional reactivity in referred youth with disruptive behavior disorders: the role of the callous-unemotional traits. Psychiatry Res. 2014;220(1-2):426-432.

4. Viding E, Fontaine NMG, Oliver BR, Plomin R. Negative parental discipline, conduct problems and callous-unemotional traits: Monozygotic twin differences study. Br J Psychiatry. 2009;195(5):414-419.

5. Eisenbarth H, Demetriou CA, Kyranides MN, Fanti KA. Stability Subtypes of Callous-Unemotional Traits and Conduct Disorder Symptoms and Their Correlates. J Youth Adolesc. 2016;45(9):1889-1901.

6. Pardini DA, Loeber R. Interpersonal callousness trajectories across adolescence: Early social influences and adult outcomes. Crim Justice Behav. 2008;35(2):173-196.

7. Kolko DJ, Dorn LD, Bukstein OG, Pardini D, Holden EA, Hart J. Community vs. clinic-based modular treatment of children with early-onset ODD or CD: a clinical trial with 3-year follow-up. J Abnorm Child Psychol. 2009;37(5):591-609.

8. American Psychiatric Association. Diagnostic and Statistical Manual of Mental Disorders, 5th Edition (DSM-5). Vol. 280; 2013.

9. Frick PJ, Ray JV, Thornton LC, Kahn RE. Annual research review: A developmental psychopathology approach to understanding callousunemotional traits in children and adolescents with serious conduct problems. J Child Psychol Psychiatry. 2014;55(6):532-548.

10. Visser JH, van der Ende J, Koot HM, Verhulst FC. Continuity of psychopathology in youths referred to mental health services. J Am Acad Child Adolesc Psychiatry. 1999;38(12):1560-1568. 
11. Fontaine NMG, Hanscombe KB, Berg MT, Mccrory EJ, Viding E. Trajectories of Callous-Unemotional Traits in Childhood Predict Different Forms of Peer Victimization in Adolescence. J Clin Child Adolesc Psychol. 2018;47(3):1-9.

12. Baskin-Sommers AR, Waller R, Fish AM, Hyde LW. Callous-Unemotional Traits Trajectories Interact with Earlier Conduct Problems and Executive Control to Predict Violence and Substance Use Among High Risk Male Adolescents. J Abnorm Child Psychol. 2015;43(8):1529-1541.

13. Klingzell I, Fanti KA, Colins OF, Frogner L, Andershed AK, Andershed H. Early Childhood Trajectories of Conduct Problems and Callous-Unemotional Traits: The Role of Fearlessness and Psychopathic Personality Dimensions. Child Psychiatry Hum Dev. 2016;47(2):236-247.

14. Salihovic S, Özdemir M, Kerr M. Trajectories of Adolescent Psychopathic Traits. J Psychopathol Behav Assess. 2014;36(1):47-59.

15. Hawes SW, Byrd AL, Waller R, Lynam DR, Pardini DA. Late childhood interpersonal callousness and conduct problem trajectories interact to predict adult psychopathy. J Child Psychol Psychiatry. 2017; 58(1):55-63.

16. Byrd AL, Hawes SW, Loeber R, Pardini DA. Interpersonal Callousness from Childhood to Adolescence: Developmental Trajectories and Early Risk Factors. J Clin Child Adolesc Psychol. 2018;47(3):1-16.

17. Ezpeleta L, Granero R, de La Osa N, Domènech JM. Developmental trajectories of callous-unemotional traits, anxiety and oppositionality in 3-7 year-old children in the general population. Pers Individ Dif. 2017;111:124-133.

18. Waller R, Baskin-Sommers AR, Hyde LW. Examining Predictors of Callous Unemotional Traits Trajectories Across Adolescence Among High-Risk Males. J Clin Child Adolesc Psychol. 2018;47(3):1-14.

19. Waller R, Shaw DS, Neiderhiser JM, et al. Toward an Understanding of the Role of the Environment in the Development of Early Callous Behavior. J Pers. 2017;85(1):90-103.

20. Fanti KA, Colins OF, Andershed H, Sikki M. Stability and change in callous-unemotional traits: Longitudinal associations with potential individual and contextual risk and protective factors. Am J Orthopsychiatry. 2017;87(1):62-75.

21. Hechtman L, Swanson JM, Sibley MH, et al. Functional Adult Outcomes 16 Years After Childhood Diagnosis of Attention-Deficit/Hyperactivity Disorder: MTA Results. J Am Acad Child Adolesc Psychiatry. 2016; 55(11):945-952.

22. Masi G, Milone A, Manfredi A, Brovedani P, Pisano S, Muratori P. Combined pharmacotherapy-multimodal psychotherapy in children with Disruptive Behavior Disorders. Psychiatry Res. 2016;238:8-13.

23. Upadhyay N, Chen H, Mgbere O, Bhatara VS, Aparasu RR. The Impact of Pharmacotherapy on Substance Use in Adolescents With AttentionDeficit/Hyperactivity Disorder: Variations Across Subtypes. Subst Use Misuse. 2017;52(10):1266-1274.

24. Waller R, Gardner F, Hyde LW. What are the associations between parenting, callous-unemotional traits, and antisocial behavior in youth? A systematic review of evidence. Clin Psychol Rev. 2013;33(4):593-608.

25. Piotrowska PJ, Stride CB, Croft SE, Rowe R. Socioeconomic status and antisocial behaviour among children and adolescents: a systematic review and meta-analysis. Clin Psychol Rev. 2015;35:47-55.

26. Pasalich DS, Dadds MR, Hawes DJ, Brennan J. Do callous-unemotional traits moderate the relative importance of parental coercion versus warmth in child conduct problems? An observational study. $J$ Child Psychol Psychiatry. 2011;52(12):1308-1315.

27. Barker ED, Oliver BR, Viding E, Salekin RT, Maughan B. The impact of prenatal maternal risk, fearless temperament and early parenting on adolescent callous-unemotional traits: a 14-year longitudinal investigation. J Child Psychol Psychiatry. 2011;52(8):878-888.

28. Muratori P, Lochman JE, Manfredi A, et al. Callous unemotional traits in children with disruptive behavior disorder: Predictors of developmental trajectories and adolescent outcomes. Psychiatry Res. 2016;236:35-41.

29. Pardini DA, Lochman JE, Powell N. The development of callousunemotional traits and antisocial behavior in children: are there shared and/or unique predictors? J Clin Child Adolesc Psychol. 2007; 36(3):319-333.
30. Masi G, Milone A, Brovedani P, Pisano S, Muratori P. Psychiatric evaluation of youths with Disruptive Behavior Disorders and psychopathic traits: A critical review of assessment measures. Neurosci Biobehav Rev. 2018;91:21-33.

31. Kimonis ER, Fanti KA, Frick PJ, et al. Using self-reported callousunemotional traits to cross-nationally assess the DSM-5 'With Limited Prosocial Emotions' specifier. Journal of Child Psychology and Psychiatry. 2015;56(11):1249-1261.

32. Pj F, Te M. A proposal to the DSM-V childhood disorders and the $\mathrm{ADHD}$ and disruptive behavior disorders work groups to include a specifier to the diagnosis of conduct disorder based on the presence of callousunemotional traits. Eur Child Adolesc Psychiatry. 2010;20:89-93.

33. Wechsler D. Wechsler intelligence scale for children. Third ed. San Antonio: TX Psychol Corp; 1991.

34. Kaufman J, Birmaher B, Brent D, et al. Schedule for Affective Disorders and Schizophrenia for School-Age Children-Present and Lifetime Version (K-SADS-PL): initial reliability and validity data. $J$ Am Acad Child Adolesc Psychiatry. 1997;36(7):980-988.

35. Hollingshead AB, Redlich FC, Class S. Mental Illness: Community Study. New York, NY, US: John Wiley \& Sons, Inc; 1958.

36. American Psychiatric Association. DSM-IV; 2000.

37. Ciucci E, Baroncelli A, Golmaryami FN, Frick PJ. The Emotional Correlates to Callous-Unemotional Traits in Children. J Child Fam Stud. 2015;24(8):2374-2387.

38. Jy W, Kuban KCK, Allred E. Manual for the ASEBA School-Age Forms and Profiles. J Child Neurol. 2001;40(4):791-799.

39. Shelton KK, Frick PJ, Wootton J. Assessment of parenting practices in families of elementary school-age children. J Clin Child Psychol. 1996; 25(3):317-329.

40. Hinshaw SP, Owens EB, Wells KC, et al. Family processes and treatment outcome in the MTA: negative/ineffective parenting practices in relation to multimodal treatment. J Abnorm Child Psychol. 2000; 28(6):555-568.

41. Pentz MA, Johnson A, Dwyer JH, Mackinnon DM, Hansen WB, Flay BR. A comprehensive community approach to adolescent drug abuse prevention: effects on cardiovascular disease risk behaviors. Ann Med. 1989;21(3):219-222.

42. Mackinnon DP, Dwyer JH. Estimating Mediated Effects in Prevention Studies. Eval Rev. 1993;17(2):144-158.

43. Muthén LK, Muthén BO. Mplus statistical analysis with latent variables. User's guide. Sixth Edition. Los Angeles: Muthén and Muthén; 2010.

44. Browne MW, Cudeck R. Alternative ways of assessing model fit. In: Bollen K, Long K (editors). Testing structural equation models. Newbury Park: Sage; 1993:136-162.

45. Hu L, Bentler PM. Fit Indices in Covariance Structure Modeling: Sensitivity to Underparameterized Model Misspecification. Psychol Methods. 1998;3(4):424-453.

46. Bollen KA, Curran PJ. Latent Curve Models. In: A Structural Equation Perspective. Hoboken: Wiley-Interscience; 2006:467;745-775.

47. Dadds MR, Cauchi AJ, Wimalaweera S, Hawes DJ, Brennan J, Outcomes BJ. Outcomes, moderators, and mediators of empathicemotion recognition training for complex conduct problems in childhood. Psychiatry Res. 2012;199(3):201-207.

48. Lochman JE, Baden RE, Boxmeyer CL, et al. Does a booster intervention augment the preventive effects of an abbreviated version of the coping power program for aggressive children? J Abnorm Child Psychol. 2014;42(3):367-381.

49. Muratori P, Bertacchi I, Giuli C, et al. First adaptation of coping power program as a classroom-based prevention intervention on aggressive behaviors among elementary school children. Prev Sci. 2015;16(3):432-439.

50. Muratori P, Milone A, Manfredi A, et al. Evaluation of Improvement in Externalizing Behaviors and Callous-Unemotional Traits in Children with Disruptive Behavior Disorder: A 1-Year Follow Up Clinic-Based Study. Administration and Policy in Mental Health and Mental Health Services Research. 2017;44(4):452-462.

51. Muñoz LC, Frick PJ. The reliability, stability, and predictive utility of the self-report version of the Antisocial Process Screening Device. Scand J Psychol. 2007;48(4):299-312. 
52. Obradović J, Pardini DA, Long JD, Loeber R. Measuring interpersonal callousness in boys from childhood to adolescence: an examination of longitudinal invariance and temporal stability. J Clin Child Adolesc Psychol. 2007;36(3):276-292.

53. Rowe R, Maughan B, Moran P, Ford T, Briskman J, Goodman R. The role of callous and unemotional traits in the diagnosis of conduct disorder. J Child Psychol Psychiatry. 2010;51(6):688-695.

54. Barker ED, Salekin RT. Irritable oppositional defiance and callous unemotional traits: is the association partially explained by peer victimization? J Child Psychol Psychiatry. 2012;53(11):1167-1175.

55. Wymbs BT, Mccarty CA, King KM, et al. Callous-unemotional traits as unique prospective risk factors for substance use in early adolescent boys and girls. J Abnorm Child Psychol. 2012;40(7):1099-1110.

56. Battagliese G, Caccetta M, Luppino OI, et al. Cognitive-behavioral therapy for externalizing disorders: A meta-analysis of treatment effectiveness. Behav Res Ther. 2015;75:60-71.

57. Muratori P, Milone A, Manfredi A, et al. Evaluation of Improvement in Externalizing Behaviors and Callous-Unemotional Traits in Children with Disruptive Behavior Disorder: A 1-Year Follow Up Clinic-Based Study. Adm Policy Ment Health. 2017;44(4):452-462.

58. Waschbusch DA, Carrey NJ, Willoughby MT, King S, Andrade BF. Effects of methylphenidate and behavior modification on the social and academic behavior of children with disruptive behavior disorders: the moderating role of callous/unemotional traits. J Clin Child Adolesc Psychol. 2007;36(4):629-644.
59. Kochanska G, Barry RA, Aksan N, Boldt LJ. A developmental model of maternal and child contributions to disruptive conduct: the first six years. J Child Psychol Psychiatry. 2008;49(11):1220-1227.

60. Fontaine NM, Rijsdijk FV, Mccrory EJ, Viding E. Etiology of different developmental trajectories of callous-unemotional traits. J Am Acad Child Adolesc Psychiatry. 2010;49(7):656-664.

61. Pardini DA, Hawes SW, Waller R, Pardini DA. Familial influences on the development of serious conduct problems and delinquency. Theory, Research and Practical Applications. In: The Development of Criminal and Antisocial Behavior; 2015:201-220.

62. Allen ML, Garcia-Huidobro D, Porta C, et al. Effective Parenting Interventions to Reduce Youth Substance Use: A Systematic Review. Pediatrics. 2016;138(2):e20154425-21.

63. Kimonis ER, Frick PJ, Cauffman E, Goldweber A, Skeem J. Primary and secondary variants of juvenile psychopathy differ in emotional processing. Dev Psychopathol. 2012;24(3):1091-1103.

64. Hawes DJ, Price MJ, Dadds MR. Callous-unemotional traits and the treatment of conduct problems in childhood and adolescence: a comprehensive review. Clin Child Fam Psychol Rev. 2014;17(3):248-267.

\section{Publish your work in this journal}

Neuropsychiatric Disease and Treatment is an international, peerreviewed journal of clinical therapeutics and pharmacology focusing on concise rapid reporting of clinical or pre-clinical studies on a range of neuropsychiatric and neurological disorders. This journal is indexed on PubMed Central, the 'PsycINFO' database and CAS,

\section{Dovepress}

and is the official journal of The International Neuropsychiatric Association (INA). The manuscript management system is completely online and includes a very quick and fair peer-review system, which is all easy to use. Visit http://www.dovepress.com/testimonials.php to read real quotes from published authors. 